\title{
Intracoronary Shear Stress and CT Characteristics of Vulnerable Coronary Plaques
}

\author{
Annabella Benedek \\ University of Medicine and Pharmacy, Tîrgu Mureș, Romania
}

\section{CORRESPONDENCE}

\section{Annabella Benedek}

Str. Gheorghe Marinescu nr. 38

540139 Tîrgu Mureș, Romania

Tel: +40 742084369

E-mail: annabell.benedek@yahoo.com

\section{ARTICLE HISTORY}

Received: July 10, 2017

Accepted: August 15, 2017

\begin{abstract}
Vulnerable coronary plaques are associated with a significant risk for rupture, and the ability to detect their characteristic features is of extreme importance, as timely detection of rupture-prone plaques could lead to the appropriate initiation of adequate therapeutic measures and prevent the evolution to an acute coronary event. The most common features of vulnerability in coronary plaques are represented by the presence of low density atheroma, a thin fibrous cap, spotty calcifications, and positive remodeling. However, there is still a huge amount of information to be learned about the role of local forces, represented by the shear stress, on the plaque vulnerability. This clinical update aims to present the most recent advances in the field of knowledge regarding the relation between shear stress and plaque vulnerability, starting from the hypothesis that shear stress significantly correlates with the $\mathrm{CT}$ features of plaque vulnerability and can represent a new marker of vulnerability in coronary artery plaques.
\end{abstract}

Keywords: shear stress, computed tomography angiography, plaque vulnerability

Recently, noninvasive diagnostic methods, especially the computed tomography (CT) angiography, have received an increasingly important role in the prevention of cardiac diseases. The role of $\mathrm{CT}$ is even more crucial in the determination of CT-based plaque-vulnerability factors. The most important CT vulnerability markers at this time are the volume of low density atheroma, the plaque burden, the thin fibrous cap, and the positive remodeling. ${ }^{1-4}$

So the main question today remains: is it possible to define a new vulnerability marker based on the CT images, and, more importantly, would it be possible for shear stress to become that new vulnerability marker?

It is known that shear stress plays an important role in the development of atherosclerosis, ${ }^{5-7}$ through complex processes that are more and more better known with the help of computer fluid dynamics. ${ }^{8-12}$

There were also several successful attempts of finding the correlation between shear stress and other vulnerability factors, defined by the use of invasive imag- 
ing devices, such as optical coherence tomography (OCT), intravascular ultrasound (IVUS), and angiography. ${ }^{13-16}$

However, to our best knowledge, no study has demonstrated so far a correlation between CT reconstructionbased shear stress values and plaque vulnerability.

\section{SHEAR STRESS DETERMINATION USING COMPUTATIONAL MODELING}

After the visual assessment, which represents the basis of the classification, the first step is the CT image-based plaque analysis, resulting in determination of the percentage of the different plaque components, which can be: dense calcium, fibrous, fibrous fatty, necrotic core volume, and undefined volume.

The next step is the 3D reconstruction, based on the data provided by the post-processing software (the diameter values of the manually selected vascular segment, which, besides the lesion itself, also included a few $\mathrm{cm}$ of the proximal and distal parts), being mainly limited on the affected region of the coronary artery, which represents one of the main limitations of this technique. ${ }^{17,18}$

Another limitation of the technique is that it is based on a less realistic approach of the reconstruction of only part of the affected coronary artery (basically, circle-shaped forms put on top of each other), thus the fluid dynamics simulation being also less realistic.

\section{SHEAR STRESS AND PLAQUE VULNERABILITY}

It has been demonstrated that vulnerable plaques are associated with a lower shear stress at the site of the lesion, a high shear stress being considered rather a protective factor against plaque development. However, plaque rupture occurs at the site of the plaque which exhibits an increased shear stress. This proves that high shear stress acting on the thin fibrous cap of the vulnerable plaque could determine the rupture of the plaque at this level. Therefore, a careful interpretation of data related to shear stress should take into consideration not only the value of shear stress, but also the location of its action. Vulnerability markers easily identified with the help of current imaging techniques include the presence of a necrotic core or a low density atheroma, positive remodeling (which actually results from exposure and adaptation to modifications of the shear stress and parietal stress acting at the site of the plaque), the presence of spotty calcifications, and the plaque volume or plaque burden. All these features have been proved to be associated with modifications in shear stress; however, the ex- act interrelation between them has not been elucidated so far. It is not clear in this moment if a decreased shear stress leads directly to an increased expression of local vulnerability markers, or both of them are the result of an exacerbation of systemic inflammation in a vulnerable patient.

Limitations of the computational determination of shear stress

The most important limitation of this technique is represented by the reconstruction method. A more realistic approach - the 3D reconstruction of the coronary artery tree - would most probably give better shear stress results, especially proximally from the plaque, the fluid dynamics simulation also being more accurate in that case.

The other setback is that, generally, in the case of hemodynamic simulations, the blood is considered a Newtonian fluid, which is, obviously, not the case in reality. Being a non-Newtonian fluid also implies that its viscosity values are affected by a number of different factors, such as blood flow velocity and the hematocrit value, which are not included in the simulation.

On the other hand, when the reconstruction is limited to the plaque-affected region of the artery, it is obvious that the proximal values of the shear stress are less reliable; however, the distal values are more suggestive and significant. A 3D reconstruction method would significantly improve the reliability of this technique for shear stress calculation.

\section{FUTURE DEVELOPMENTS OF COMPUTATIONAL FLUID DYNAMICS FOR SHEAR STRESS ASSESSMENT}

The next step for advancing this technique is planned to be the more realistic $3 \mathrm{D}$ reconstruction of the coronary tree, which will enable a better approximation of the shear stress values along the plaque, and also proximally and distally from it. In our team, we will also try to use the viscosity-modifying factors in the hemodynamic simulations, most importantly the hematocrit value and the blood flow velocity.

Another further development could be the fusion of CT images with other images (angiography, OCT, IVUS), which would allow a more accurate reconstruction of the coronary tree.

\section{CONFLICT OF INTEREST}

Nothing to declare. 


\section{REFERENCES}

1. Nyulas T, Chitu M, Mester A, et al. Computed tomography biomarkers of vulnerable coronary plaques. Journal of Interdisciplinary Medicine. 2016:1:263-266.

2. Benedek T, Jako B, Benedek I. Plaque quantification by coronary CT and intravascular ultrasound identifies a low CT density score as a marker of plaque instability in acute coronary syndromes. Int Heart J. 2014;55:22-28.

3. Maurovich-Horvat $P$, Ferencik M, Voros S, Merkely B, Hoffmann U. Comprehensive plaque assessment by coronary CT angiography. Nat Rev Cardiol. 2014;11:390-402.

4. Schaar JA, Muller JE, Falk E, et al. Terminology of high-risk and vulnerable coronary artery plaques. Eur Heart J. 2004;24:1077-1082.

5. Ross R. Atherosclerosis is an inflammatory disease. Am Heart $\mathrm{J}$. 1999;138:S419-S420.

6. Dirksen MT, van der Wal AC, van der Berg FM, van der Loos CM, Becker $A E$. Distribution of inflammatory cells in atherosclerotic plaques relates to the direction of flow. Circulation 1998:98:2000-2003.

7. Traub $\mathrm{O}$, Berk BC. Laminar shear stress: mechanisms by which endothelial cells transduce an atheroprotective force. Arterioscler Thromb Vasc Biol. 1998 May;18(5):677-85.

8. Steiman DA. Image-based computational fluid dynamics modeling in realistic arterial geometries. Ann Biomed Eng. 2002;30:483-497.

9. Sun Z, Xu L. Computational fluid dynamics in coronary artery disease. Comput Med Imaging Graph. 2014;38:651-663.

10. Papaioannou TG, Stefanadis C. Vascular wall shear stress: basic principles and methods. Hellenic J Cardiol. 2005:46:9-15.
11. Walpola PL, Gotlieb Al, Langille BL. Monocyte adhesion and changes in endothelial cell number, morphology, and F-actin distribution elicited by low shear stress in vivo. Am J Pathol. 1993:142:1392-1400.

12. Davies PF. Flow-mediated endothelial Mechanotransduction. Physio/ Rev 1995:75:519-560

13. van der Giessen A, Schaap M, Gijsen FJ, et al. 3D fusion of intravascular ultrasound and coronary computed tomography for in-vivo wall shear stress analysis: a feasibility study. Int J Cardiovasc Imaging. 2010;26:781 796.

14. Stone PH, Saito S, Takahashi S, et al. Prediction of progression of coronary artery disease and clinical outcomes using vascular profiling of endothelial shear stress and arterial plaque characteristics, the PREDICTION study. Circulation. 2012;126:172-181.

15. Huang D, Muramatsu T, Li Y, et al. Assessment of endothelial shear stress in patients with mild or intermediate coronary stenoses using coronary computed tomography angiography: comparison with invasive coronary angiography. Int J Cardiovasc Imaging. 2017;33:1101-1110.

16. Ohayon J, Finet G, Treyve F, Rioufol G, Dubreuil O. A three-dimensiona finite element analysis of stress distribution in a coronary atherosclerotic plaque: in-vivo prediction of plaque rupture location. Biomechanics Applied to Computer Assisted Surgery. 2005:225-241.

17. Giannopoulos AA, Chatzizisis YS, Maurovich-Horvat P, et al. Quantifying the effect of side branches in endothelial shear stress estimates. Atherosclerosis. 2016;251:213-218.

18. Stone $\mathrm{PH}$, Coskun $\mathrm{AU}$, Prati F. Ongoing methodological approach to improve the in vivo assessment of local coronary blood flow and endothelial shear stress. J Am Coll Cardiol. 2015;66:136-138. 